29

30

31

32

33

34

35

36

37

38

39

40

41

42

\title{
Reliability of the 505 Change of Direction Test in Netball Players
}

Submission Type: Original Investigation

Olivia, R. Barber, Christopher Thomas, Paul, A. Jones, John, J. McMahon \& Paul Comfort ${ }^{\#}$

Human Performance Laboratory, Directorate of Sport, Exercise and Physiotherapy, University of Salford, Salford, Greater Manchester. M6 6PU. United Kingdom

\#Corresponding Author: Paul Comfort - p.comfort@ salford.ac.uk

Preferred running head: Reliability of 505 Change of Direction

Abstract word count: 246 words

Manuscript word count: 2330 words

Number of tables and figures: 1 Table, 1 Figure 
43

44

45

46

47

48

49

50

51

52

53

54

55

56

57

58

59

60

61

62

63

64

65

66

67

68

69

70

71

72

73

74

75

\section{Abstract}

Purpose: To determine the reliability of the 505 change of direction (COD) test performed with both a stationary and flying start. Methods: Fifty-two female netball players (age $23.9 \pm$ $5.4 \mathrm{yrs}$, height $169.9 \pm 3.3 \mathrm{~cm}$, body mass $65.2 \pm 4.6 \mathrm{~kg}$ ) performed 6 trials of the $505 \mathrm{COD}$ test, three with a flying start and three with a stationary start, once per week over a four week period, to determine within- and between-session reliability. Results: Testing revealed high within-session reliability for the stationary start $(\mathrm{ICC}=0.96-0.97)$ and for the flying start $($ ICC $=0.90-0.97)$. Similarly, both the stationary start $($ ICC $=0.965)$ and the flying start demonstrated high reliability $(\mathrm{ICC}=0.951)$ between-sessions, although repeated measures analysis of variance $(\mathrm{p}<0.001)$ revealed learning effects were found to be present betweensessions for both tests. Performances stabilized on the second day for the static start and on the third day of testing for the flying start. Conclusions: Results suggest that the 505 COD test is a reliable test in female netball players, with either a stationary or flying start. Smallest detectable differences of $3.91 \%$ and $3.97 \%$ for the stationary start and the flying start, respectively, allow practitioners to interpret whether changes in time taken to complete the 505 COD test reflect genuine improvements in performance or are measurement errors. It is suggested that one day of familiarization testing is performed for the stationary start and two days of familiarization for the flying start, to minimize learning effects.

Key Words: Agility; Learning affects; Smallest Detectable Difference; Meaningful

1 Difference

2


78 The importance of change of direction (COD) and agility has been highlighted in many team 79 sports, including football, ${ }^{1}$ rugby, ${ }^{2}$ basketball, ${ }^{3}$ volleyball, ${ }^{4}$ and netball. ${ }^{5-8}$ Emphasising 80 high speed movements may only contribute to a low percentage of match distance covered, but they are crucial to many match winning situations, such as gaining possession and preventing or creating scoring opportunities. ${ }^{1,7,8}$ During elite netball matches in particular, athletes can perform up to $81.3 \pm 20.1$ high-intensity sprints ${ }^{6}$ and $63.7 \pm 7.6$ COD maneuvers, ${ }^{5}$ which highlights the importance of these tasks to competitive netball performance and warrants the inclusion of agility/COD assessments within netball performance testing batteries. Agility tests are widely used within sports testing batteries to establish an athlete's ability to rapidly change direction. ${ }^{3,9}$ Although many tests are referred to as agility tests, they are usually methods of assessing COD performance, as agility includes a reaction to a stimulus, which is not part of the majority of these tests. ${ }^{9}$ Many COD tests, such as the Illinois agility run, $505 \mathrm{COD}$ test and T-Test, attempt to mimic common movement patterns performed within a given sport, ${ }^{10}$ however, few studies have investigated

92 the reliability of these tests. ${ }^{2,11,12}$ Reliability of methods of assessment is highly important to ensure that sports scientists and researchers can appropriately interpret changes in performance as being meaningful or a product of the error inherent within the testing procedures adopted.

97 Research has reported that within-session learning effects are present during COD t-tests, but 98 this stabilizes after only one trial. ${ }^{11}$ The 505 COD test has also been shown to yield a reliable measure of COD amongst female softball players, with a high test-retest reliability (intra100 class correlation coefficient (ICC) $\geq 0.93$ ), ${ }^{12}$ although learning effects were not reported. 
101 Typically, the protocol for the 505 COD test allows a ten meter run up (flying start) before 102 crossing the start line and timing commencing. Although the reliability of the 505 COD test

103 has been investigated previously, ${ }^{12}$ no studies have specifically assessed female netball 104 players or compared the reliability of stationary and flying starts, or reported the potential 105 learning affects during both tests. Identification of any systematic learning effects are 106 essential to ensure that sports scientists and researchers apply appropriate methods when 107 collecting baseline data, to ensure that any subsequent changes in performance are meaningful and are not due to learning effects.

110 The purpose of this study, therefore, was to assess the within- and between-session reliability 111 of the 505 COD test performed with both stationary and flying (ten meter approach) starts. It 112 was hypothesized that both tests would demonstrate a learning effect, with improved 113 performances between the initial sessions; and that the stationary start for the 505 COD test 114 would be the most reliable as it is easier to standardize. A further aim was to identify the 115 smallest detectable differences in performances between-sessions to aid practitioners in 116 determining meaningful changes in 505 COD test performances.

119 Methods

\section{Experimental Design}

122 This study assessed the within- and between-session reliability of the 505 COD test to 123 identify the magnitude of difference which reveals a meaningful change in performance. A 124 secondary aim was to determine if learning effects were present in trained female netball 125 players who had no previous experience of performing the 505 COD test. Previous studies 
126 have typically assessed the between-session reliability of COD tests over two to three

127 sessions ${ }^{11,13}$ and have indicated that the magnitude of observed learning effects is dependent

128 upon both the number of trials and the task being performed. The COD tests included in the

129 present study were, therefore, completed on the same day each week for four weeks, at the

130 same time of day (19:00-20:00), where subjects performed six repetitions of the 505 COD

131 test; three with a flying start and three with a stationary start. The same researcher and the

132 coaching staff were present at all sessions to ensure that a similar level of athlete motivation

133 was achieved between-sessions. This approach allowed within- and between-session

134 reliability and measurement error to be calculated and learning effects to be determined.

135 Within-session reliability was determined using the ICC, standard error of measurement

136 (SEM), smallest detectable difference (SDD), and 95\% confidence intervals. Repeated-

137 measures analysis of variance (RMANOVA) was used to assess between-session reliability

138 and learning effects.

140 Subjects

141 Fifty-two female players (age $23.9 \pm 5.4$ yrs, height $169.9 \pm 3.3 \mathrm{~cm}$, body mass $65.2 \pm 4.6$

$142 \mathrm{~kg}$, average playing experience $14.8 \pm 4.9 \mathrm{yrs})$ volunteered to participate in this study. All

143 subjects were injury free and had $\geq$ five years experience of playing netball for a minimum of

144 one hour $\geq$ two $x$ week. All participants provided written informed consent to participate, and

145 the University of Salford Research and Ethics Committee approved the research and 146 conformed to the Code of Ethics of the World Medical Association (Declaration of Helsinki).

$148 \quad$ Procedures

149 The 505 COD test requires subjects to sprint five meters, turn $180^{\circ}$ and sprint a further five 150 meters (Figure 1). A 'flying start' allowed the subject a $10 \mathrm{~m}$ run up before crossing the start 
151 line and timing commenced. A 'stationary start' required a static start position $0.5 \mathrm{~m}$ behind 152 the start line, to prevent early triggering of the timing gates. Subjects were asked to plant their 153 dominant foot upon executing the turn.

Testing took place once a week, at the same day and time (19:00-20:00), on the same netball court, for four weeks, at the start of the competitive season. After a standardized progressive warm up, participants performed three timed attempts with both stationary and flying starts (six trials in total, per session). All times were recorded using Brower timing gates (Brower, Speed Trap 2 Wireless Timing System, UT, USA) extended to approximately hip height. The time started when a participant first passed through the timing gates and stopped when the participant passed through them again upon their return. One minute of recovery time was given between each attempt, with a three minute rest period prescribed between the flying and static starts. Participants were requested to standardize their dietary intake during each day of testing and to avoid strenuous exercise for the 48 hours prior to testing.

Statistical Analyses

172 Statistical analysis was performed using SPSS version 20.0 (IBM, USA). Descriptive 173 statistics (mean, standard deviation (SD) and 95\% confidence intervals) were calculated for 174 time to complete the 505 COD test. Within-session reliability was determined using the ICC, 175 and interpreted following the criteria of: Poor $=0.40 ;$ Fair $=0.40-0.70$; Good $=0.70-0.90$; 
176 and Excellent $=0.90 .^{14}$ Between-session reliability was determined, using the best

177 performances from each day, via ICCs and two separate (static and flying 505 COD tests)

178 repeated measures ANOVA (RMANOVA), with Bonferroni post hoc analysis. An apriori

179 alpha level was set at $\mathrm{p} \leq 0.05$. Effect sizes were also measured using partial Eta squared, to

180 determine the magnitude of difference between days, and interpreted according to the Cohen

$181 d$ method, ${ }^{15}$ which defines $0.2,0.5$, and 0.8 as small, medium and large, respectively. The

182 SEM was calculated from the formula $((\operatorname{SD}$ (pooled) $\mathrm{x}(\sqrt{1} 1-\mathrm{ICC}))$, and the $\mathrm{SDD}$ was

183 calculated using the formula $(1.96 \times \sqrt{ } 2) \mathrm{SEM}){ }^{11}$

184

185 Results

186 Within-Session Reliability

187 The ICCs for both the stationary 505 (0.96-0.97) and flying $505(0.90-0.97)$ showed excellent within-session reliability (Table 1).

\section{Between-Session Reliability and Learning affects}

191 The ICCs for both the stationary 505 (0.968) and flying $505(0.951)$ also showed excellent reliability between-sessions. The RMANOVA identified significant differences between days for the stationary start $\left[\mathrm{F}_{(3,153)}=9.031, p<0.001, \eta=0.22\right.$, power 0.96$]$, with Bonferroni posthoc analysis identifying that 505 performances from a static start on days two, three and four were significantly faster $(2.84 \pm 0.22 \mathrm{~s}, 2.84 \pm 0.23 \mathrm{~s}, 2.82 \pm 0.22 \mathrm{~s}, \mathrm{p} \leq 0.01$, respectively $)$ when compared to day one $(2.88 \pm 0.23 \mathrm{~s})$. There were no significant differences $(\mathrm{p}>0.05)$ between days two, three or four (Table 1).

199 Similarly, there was a significant difference between days for the 505 performed with a flying 200 start $\left[\mathrm{F}_{(3,153)}=2.319, p<0.01, \eta=0.04\right.$, power 0.95$]$, with post-hoc analysis for the flying 505 
identifying significantly faster performances on days three and four $(2.54 \pm 0.16 \mathrm{~s}, 2.52 \pm$ $0.17 \mathrm{~s}, \mathrm{p} \leq 0.01$, respectively) compared to day one $(2.57 \pm 0.18 \mathrm{~s})$. There were no additional significant differences ( $\mathrm{p}>0.05)$ between testing days (Table 1$)$.

[***Insert Table 1 about here***]

\section{Discussion}

211 The results of this study demonstrated a high within-session and between-session reliability 212 (ICC $\geq 0.899$ ) for both versions of the 505 COD test; however, both tests did demonstrate learning effects between-sessions, in line with our hypotheses. Performance in the 505 performed with a static start stabilizes after one day of familiarization, whereas the flying 505 appears to stabilize after two days of familiarization in female netball players.

The administration of COD testing, in particular the 505 COD test, is popular in team-sports 12, 16, 17; however, it is important that the 505 COD test demonstrates high reliability so results can be interpreted appropriately. Therefore, practitioners should be are aware of the learning effects of each test. Within-session reliability of the 505 COD test demonstrated excellent reliability ( $\mathrm{ICC}=0.959-0.974)$, with the exception of the flying start on day one which only demonstrated a good reliability score (ICC $=0.899)$. A previous study also investigating female athletes reported similar reliability $(\mathrm{ICC}=0.92)$, in line with our findings. ${ }^{12}$

There was evidence of a learning effect with significant differences $(p<0.001)$ present between testing sessions for the stationary start and flying start. With a stationary start, days two, three and four all resulted in small but significantly $(\mathrm{p}<0.01)$ faster times compared to day one, although there were no differences between days two, three and four. These results demonstrate that only one day of familiarization is required for performances to stabilize during the 505 COD test performed from a stationary start. In addition, with a flying start, the only significant differences $(\mathrm{p}<0.01)$ were found between day one and days three and four, 
with no significant difference $(\mathrm{p}>0.05)$ between days three and four, highlighting that performances stabilized after two days of familiarization for the flying 505 COD test. It can be concluded, therefore, that the 505 COD test is a reliable test, when performed with either a stationary or flying start, although some familiarization is required. The difference in familiarization required between the stationary start and the flying start may be attributable to the fact that running velocity and therefore momentum is likely to be higher during the flying start. The flying start also adds the potential for additional variability in the approach velocity and therefore slightly reduces the reliability of the test. It is suggested that future research determine the variability and effect of approach speed on the reliability and performance in the flying 505 COD test.

The presence of learning effects during the administration of the 505 COD test, for both stationary and flying starts highlights the need for practice trials to be administered before testing, to ensure the most reliable outcome is achieved. We suggest two practice trials are adequate, as followed in the aforementioned protocol. The excellent to good ICC scores allow coaches to administer the $505 \mathrm{COD}$ test female netball players with confidence. Munro and Herrington ${ }^{11}$ explain that SEM values show the range in which an individual's true score is likely to lie, whereas SDD values allow practitioners to interpret whether a change in an individual's performance is significant. The SEM and SDD values gained from this research will allow coaches to evaluate true changes in performance and eliminate measurement error as a cause of change. With a base of raw data being collected, if the same protocol is followed, comparisons across netball teams and between players will be made easier.

No studies, to our knowledge, have presented the SEM and SDD values for the 505 COD test, using female participants. With no statistical evidence providing measurement error values it is difficult for coaches to identify meaningful improvements, however this study demonstrates that changes of $\geq 3.91 \%$ and $\geq 3.97 \%$ for the stationary start and the flying start, respectively, in female netball players, are meaningful. It should be acknowledged that COD was only assessed for the dominant leg in this study and therefore reliability and learning effects of the 505 COD test using the non-dominant leg may be more varied. Previous research showed that flying 505 COD times of elite female softball players decreased by $5.48 \%(\mathrm{p}=0.03)$ for the non-dominant leg and by $1.09 \%(\mathrm{p}>0.05)$ for dominant leg across a competitive season. ${ }^{18}$ When applying the results of the present study to the aforementioned data, it can be reasoned that the change in flying 505 COD performance noted for the 
266 dominant leg was not meaningful which is line with the reported effect size $(d=0.43)$. It is

267

268

269

270

271

272

273

274

275

276

277

278

279

280

281

282

283

284

285

286

287

288

289

290

291

292

293

294

295

296 suggested that future research should compare performances, reliability and learning effects of the 505 COD test variations between limbs in order to establish what a meaningful change in the performance of these tasks with the non-dominant is for future studies and to allow for a more accurate interpretation of previous findings.

\section{Practical Applications}

It is recommended that strength and conditioning coaches ensure appropriate familiarization with the 505 COD test prior to testing athletes, consisting of one familiarization session for the 505 COD performed with a stationary start and two familiarizations sessions if performed with a flying start. Additionally, differences in 505 COD times of $\geq 3.91 \%$ and $\geq 3.97 \%$ for the stationary start and the flying start, respectively, in female netball players, highlight meaningful changes. Future research should seek to determine if the level of reliability and learning effects are similar in other team sports.

\section{Conclusion}

The results of this study demonstrate a high within-session and between-session reliability for both versions of the 505 COD test. Both tests do, however, demonstrate learning effects between-sessions. Performance in the 505 performed with a static start stabilizes after one day of familiarization, whereas the flying 505 appears to stabilize after two days of familiarization, however, it should be noted that these changes between-sessions, while statistically significant, were small.

Acknowledgements: The authors would like to thank each of the subjects for their participation. The results of the current study do not constitute endorsement of the product by the authors or the journal 
298

299

300

301

302

303

304

305

306

307

308

309

310

311

312

313

314

315

316

317

318

319

320

321

322

323

324

325

326

327

328

329

330

331

332

333

334

335

336

337

338

339

340

341

342

1. Little T, Williams AG. Specificity of acceleration, maximum speed, and agility in professional soccer players. J Strength Cond Res. Feb 2005;19(1):76-78.

2. Gabbett TJ, Kelly JN, Sheppard JM. Speed, change of direction speed, and reactive agility of rugby league players. J Strength Cond Res. Jan 2008;22(1):174-181.

3. Spiteri T, Nimphius S, Hart NH, Specos C, Sheppard JM, Newton RU. Contribution of strength characteristics to change of direction and agility performance in female basketball athletes. J Strength Cond Res. Sep 2014;28(9):2415-2423.

4. Gabbett T, Georgieff B. Physiological and anthropometric characteristics of Australian junior national, state, and novice volleyball players. J Strength Cond Res. Aug 2007;21(3):902-908.

5. Fox A, Spittle M, Otago L, Saunders N. Offensive Agility Techniques Performed During International Netball Competition. International Journal of Sports Science \& Coaching. 2014;9(3):543-552.

6. Fox A, Spittle M, Otago L, Saunders N. Activity profiles of the Australian female netball team players during international competition: Implications for training practice. Journal of Sports Sciences. 2013;31(14):1588-1595.

7. Chandler PT, Pinder SJ, Curran JD, Gabbett TJ. Physical Demands of Training and Competition in Collegiate Netball Players. The Journal of Strength \& Conditioning Research. 2014;28(10):2732-2737.

8. Davidson A, Trewartha G. Understanding the Physiological Demands of Netball: a time-motion investigation. International Journal of Performance Analysis in Sport. 2008;8(3):1-17.

9. Sheppard J, Young W. Agility literature review: Classifications, training and testing. Journal of Sports Sciences. 2006;24(9):919-932.

10. Otago L. A game analysis of the activity patterns of netball players. Sports Coach. 1983;7(1):24-28.

11. Munro AG, Herrington LC. Between-session reliability of four hop tests and the agility T-test. J Strength Cond Res. May 2011;25(5):1470-1477.

12. Nimphius $S$, McGuigan MR, Newton RU. Relationship between strength, power, speed, and change of direction performance of female softball players. J Strength Cond Res. Apr 2010;24(4):885-895.

13. Stewart PF, Turner AN, Miller SC. Reliability, factorial validity, and interrelationships of five commonly used change of direction speed tests. Scand J Med Sci Sports. Jun 2012;24(3):500-506.

14. Cortina JM. What is Coefficient Alpha? An Examination of Theory and Applications. . Journal of Applied Psychology. 1993;38(1):98-104.

15. Thomas JR, Nelson JK, Silverman SJ. Research Methods in Physical Activity. Champaign, IL: Human Kinetics; 2005.

16. Gabbett TJ. A comparison of physiological and anthropometric characteristics among playing positions in sub-elite rugby league players. J Sports Sci. Dec 2006;24(12):1273-1280.

17. Young WB, Miller I, Talpey S. Physical qualities predict change-of-direction speed but not defensive agility in Australian Rules football. The Journal of Strength \& Conditioning Research. 2015;29(1):206-212. 
363

364

365

366

367

368

369

370

371

372

373

374

375

376

377

378

379

380

381

382

383

384

385

386

387

388

389

390

391

392
18. Nimphius S, McGuigan MR, Newton RU. Changes in Muscle Architecture and Performance During a Competitive Season in Female Softball Players. The Journal of Strength \& Conditioning Research. 2012;26(10):2655-2666. 
Table and Figure Legends

394 Table.1. Comparison of performances (Means \pm SD, 95\% Confidence Intervals) and 395 reliability statistics (ICC, SEM and SDD) across days

\begin{tabular}{|c|c|c|c|c|c|}
\hline Trial Day & Mean \pm SD (s) & $95 \% \mathrm{CI}$ & ICC & SEM & SDD $(s)_{306}^{396}$ \\
\hline Stationary 1 & $2.88 \pm 0.23$ & $2.80-2.93$ & 0.959 & 0.05 & $0.130(4.51 \%)$ \\
\hline Stationary 2 & $2.84 \pm 0.22 *$ & $2.77-2.89$ & 0.969 & 0.04 & 0.105 (3.00\% \\
\hline Stationary 3 & $2.84 \pm 0.23^{*}$ & $2.77-2.90$ & 0.971 & 0.04 & $0.105(3.00 \%)$ \\
\hline Stationary 4 & $2.82 \pm 0.22 *$ & $2.75-2.88$ & 0.973 & 0.04 & $0.100(3.55$ \\
\hline Mean & $2.84 \pm 0.22$ & $2.78-2.90$ & 0.968 & 0.04 & $0.111(3.91 \%)$ \\
\hline Flying 1 & $2.57 \pm 0.18$ & $2.51-2.61$ & 0.899 & 0.06 & $0.16(6.23 \%)$ \\
\hline Flying 2 & $2.55 \pm 0.17$ & $2.50-2.59$ & 0.974 & 0.03 & $0.08\left(3.14_{\Delta \beta}^{O}\right.$ \\
\hline Flying 3 & $2.54 \pm 0.18 *$ & $2.49-2.59$ & 0.963 & 0.04 & $0.10(3.94 \%)$ \\
\hline Flying 4 & $2.52 \pm 0.16^{*}$ & $2.47-2.56$ & 0.966 & 0.03 & $0.08\left(3.17_{\Delta}^{\circ}\right)$ \\
\hline Mean & $2.52 \pm 0.17$ & $2.48-2.56$ & 0.951 & 0.04 & $0.10(3.97 \%)$ \\
\hline \multicolumn{6}{|c|}{ *Significantly different from Day $1(\mathrm{p} \leq 0.01)$} \\
\hline
\end{tabular}

410

411

412

413

414

415

416

417

418

419

420

421

422

423

424

425

426

427

428

429

430

431

432

433

434

435

436

437

438 

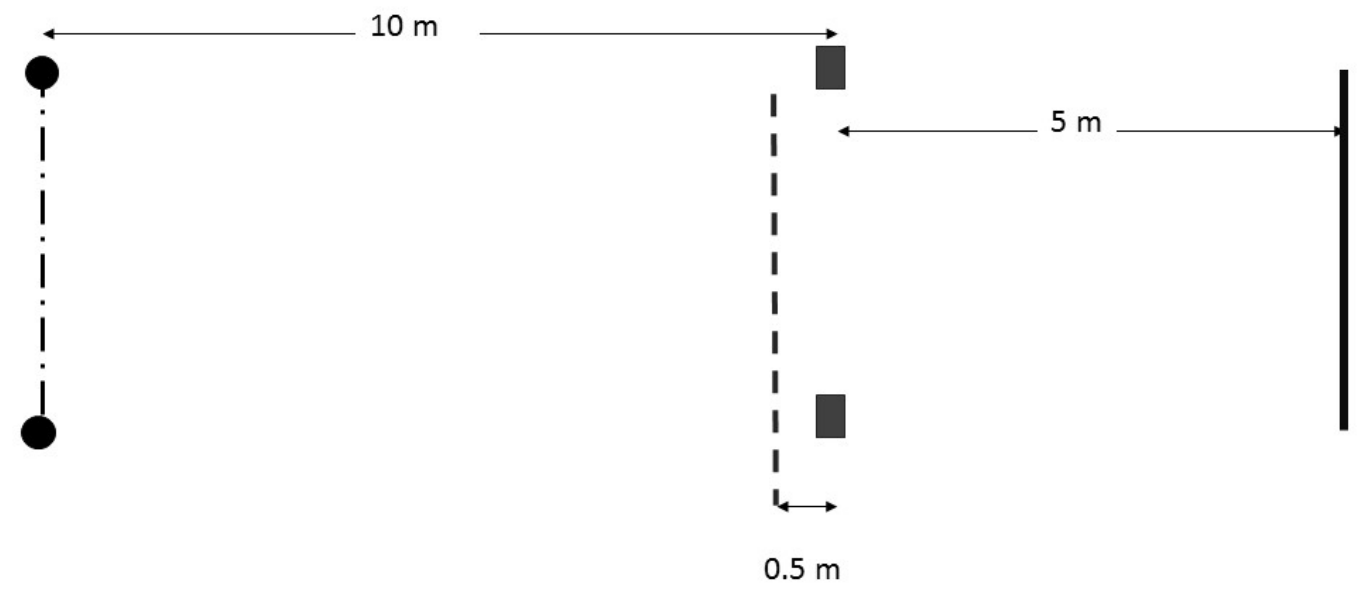

Key:

'Flying' start point $\quad-\cdot-\cdot-\cdot-$

'Stationary' start point - - - - - -

'Turning' point

439

Timing cells

Figure 1. Example of the 505 change of direction set up. 